\title{
Revealing spectral cross-correlations in radiation of multiwavelength fiber laser with randomly distributed feedback
}

I. Vatnik, O. Gorbunov, S. Sugavanam, D. Churkin

I. Vatnik, O. Gorbunov, S. Sugavanam, D. Churkin, "Revealing spectral cross-correlations in radiation of multiwavelength fiber laser with randomly distributed feedback," Proc. SPIE 10683, Fiber Lasers and Glass Photonics: Materials through Applications, 1068335 (17 May 2018); doi: 10.1117/12.2307311

SPIE. Event: SPIE Photonics Europe, 2018, Strasbourg, France 


\title{
Revealing spectral cross-correlations in radiation of multiwavelength fiber laser with randomly distributed feedback
}

\author{
I. Vatnik ${ }^{\mathrm{a}}$, O. Gorbunov ${ }^{\mathrm{a}, \mathrm{b}}, \mathrm{S}$. Sugavanam ${ }^{\mathrm{c}}, \mathrm{D}$. Churkin ${ }^{\mathrm{a}}$ \\ ${ }^{a}$ Novosibirsk State University, Pirogova str. 2, Novosibirsk 630090, Russia; ${ }^{b}$ Institute of Automation \\ and Electrometry SB RAS, Academician Koptyug ave. 1, Novosibirsk 630090, Russia; ${ }^{\mathrm{c}}$ Aston \\ Institute of Photonic Technologies, Aston University, Birmingham, B4 7ET, United Kingdom;
}

\begin{abstract}
In present paper correlations between different parts of spectrum of a fiber laser with randomly distributed feedback (RDFL) were experimentally measured directly. Implemented statistical analysis demonstrate weak cross-correlations between different lines in generation spectrum. These correlations were vizualized by plotting 2-D probability density functions. Linear correlation coefficient (Pearson coefficient) was calculated for each pair of spectrum lines.
\end{abstract}

Keywords: laser radiation statistics, fiber laser with randomly distributed feedback

\section{INTRODUCTION}

Statistics of fiber lasers' radiation is continuously studied during the last decade. General approach, used for description of statistical properties of conventional single-mode lasers cannot be directly used for multimode fiber radiation [1], generated in fiber lasers. Nonlinear interaction of numerous modes lead to complicated temporal dynamics, for which statistical description is only possible. Non-trivial properties of radiation dynamics in fiber lasers with fiber Bragg gratings was observed in numerical simulations [2] and a number of experiments in different fiber laser types [3, 4]. It was shown, that probability density function (PDF) has non-exponential shape, what indicates presence of spectral correlations. More general approach was implemented in [5] to predict general properties of radiation statistics of turbulent radiation of a fiber laser. Fiber lasers with randomly distributed feedback (RDFLs) have additional differ as there are no separate spectral modes due to absence of localized mirrors, so their radiation statistical properties are even more mysterious. Indirect observations [6] show that PDF of generated radiation has non-exponential shape, so correlations between different spectral components should exist. In present paper an attempt to measure these correlations directly was performed. Weak correlations were observed and analyzed.

\section{SIMULTANEOUS MEASUREMENT OF DIFFERENT SPECTRAL LINES IN RDFL}

As one of key problems for experimental investigation of statistical propertied of fiber laser radiation is fast decay of spectral power density during shift from spectrum center to spectrum edge a scheme of RDFL with Lyot filer (LF) was chosen. In this single armed, forward pumped configuration a LF is placed at one end of long fiber span, what results in a flat modulated spectrum, convenient for direct measurement of correlations. For more details see [7]. Experimental setup is schematically shown on fig. 1a. 

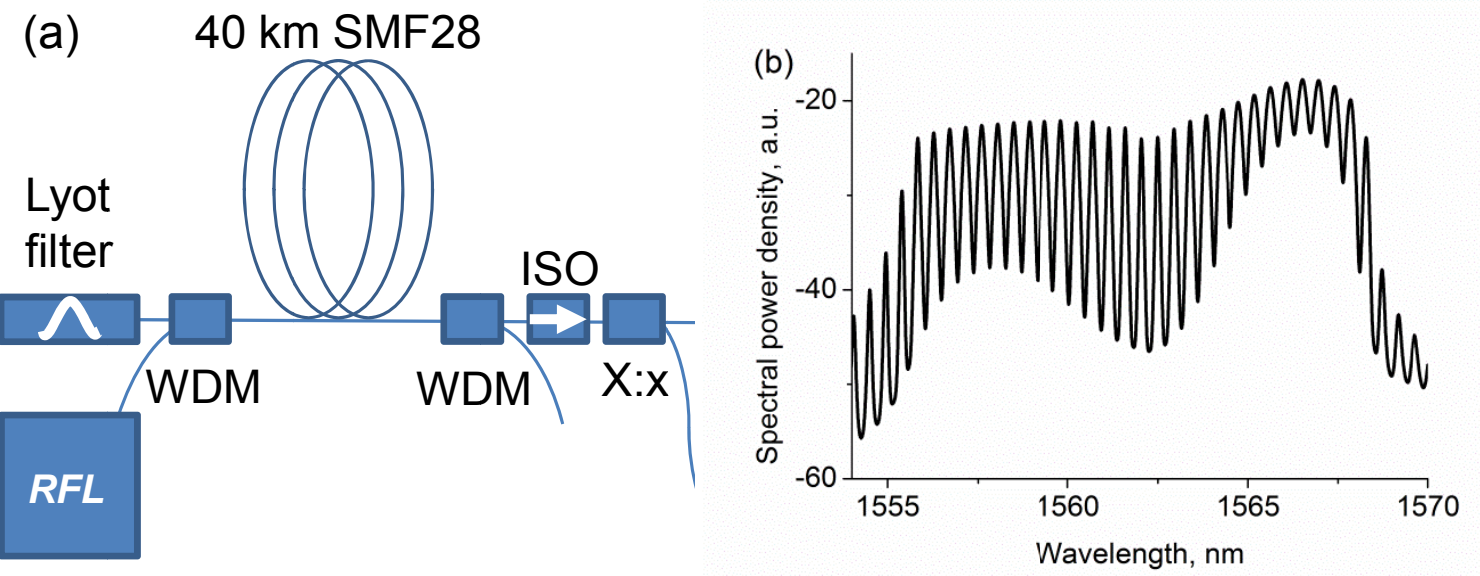

Figure 1. a) Experimental setup b) Characteristic generation spectrum of RDFL with LF

The laser cavity is formed using a $40 \mathrm{~km}$ span of Corning SMF 28 standard telecommunications fiber. A CW Raman fiber laser operating at $1455 \mathrm{~nm}$ is splice-coupled to the ultralong fiber span using a wavelength division multiplexer (WDM) as the pump source. The Lyot filter (LF) is spliced to the RFL-FL configuration near the pump end. As it works in transmission, a 50:50 coupler based fiber mirror was used to reflect the filtered radiation back. Laser output is measured at the far end of the $40 \mathrm{~km}$ fiber span. Undepleted pump power and spurious back reflections are removed by WDM and two stage isolator (return losses $>60 \mathrm{~dB}$ ) respectively.

Lasing with power of hundreds $\mathrm{mW}$ is observed at pump higher than $1.1 \mathrm{~W}$ (see details in [6]). Laser spectra well above the threshold are shown on fig. 1b; characteristic spectrum width is $13 \mathrm{~nm}$. Due to LF influence it is periodical and consist of single lines. Width of a such line is equal by order of magnitude to oscilloscope bandwidth. Lines have comparable amplitude - difference is less than $6 \mathrm{~dB}$, - so all lines have enough energy to be registered by photodetector. PDF of single lines were experimentally measured in [8]. It was shown that PDF shape of single lines depend on its position in generation spectrum: lines in center of two major peaks have PDF close to Gaussian while at spectrum edge and in dip between major peaks PDF have significantly longer tails. Performed half-qualitative analysis of their interplay during formation of the entire spectrum, which has strongly non-Gaussian statistics, have allowed to assume that separate lines cannot be treated as statistically independent and should correlate with each other.

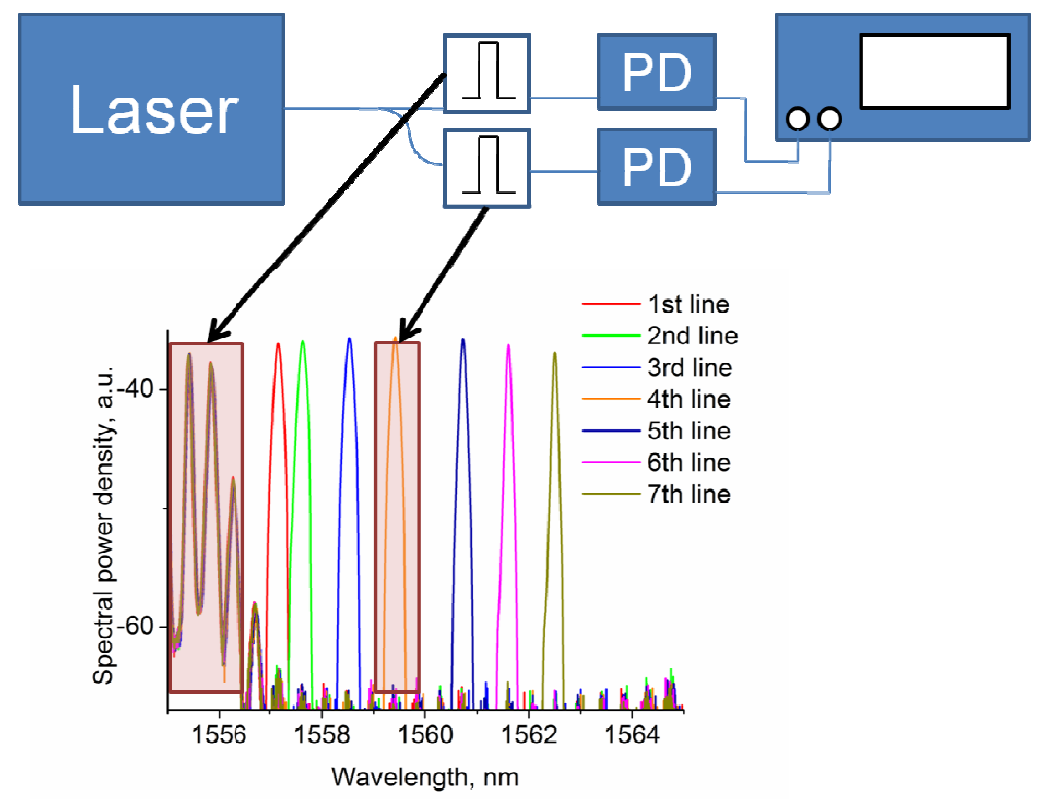

Figure 2. Technique of simultaneous measurement of two lines' temporal dynamics. 
Process of simultaneous measurements of different lines of generated laser spectrum is schematically illustrated at fig. 2. A coupler at laser output divides radiation into two channels. Each of them is passed through tunable spectral filter to cut desirable spectral line, which then are detected by two fast $(50 \mathrm{GHz})$ photodetectors, transmitting electrical signals to two channels of oscilloscope operating with bandwidth $16 \mathrm{GHz}$. In experiments position and width of one spectral filter was fixed at the edge of spectrum, while second was swept through other spectral lines of left major peak. Length of measured time dynamics was $10^{9}$ points with discretization step 12.5 ps. Totally 7 pairs of lines were measured.

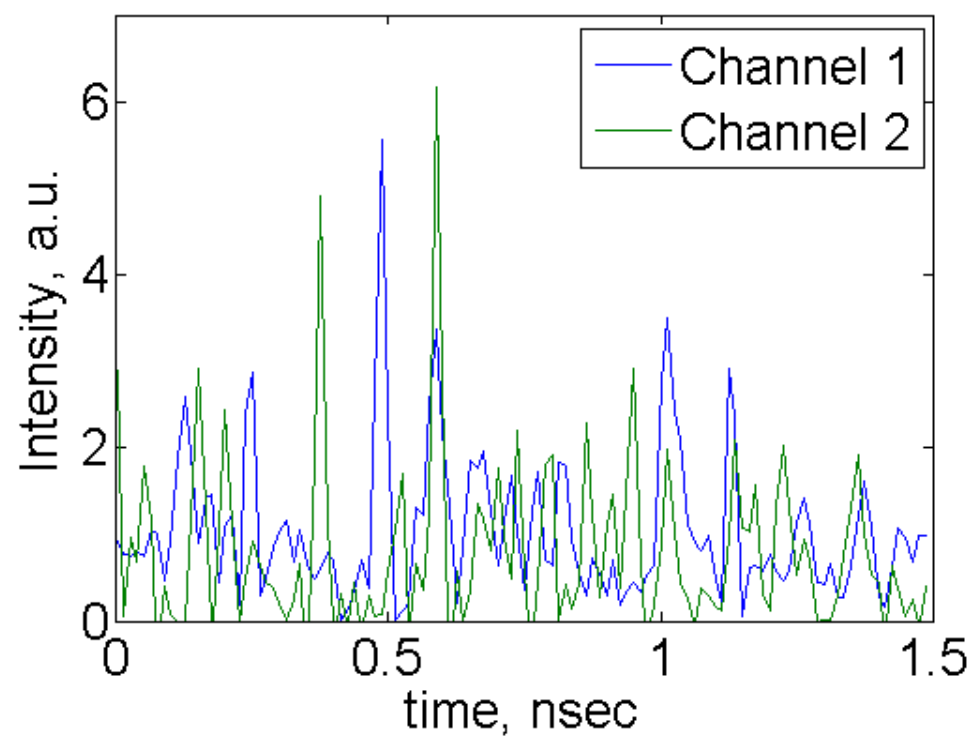

Figure 3. Characteristic temporal dynamics at two channels

Fig. 3 illustrates two simultaneous temporal dynamics from two oscilloscope channels. It is hard to notice some correlation visually, what indicates that it can be expected to appear weak, if any. But calculation of cross-correlation function $(\mathrm{CCF})$

$$
C C F(\tau)=\frac{\frac{1}{T} \int I_{1}(t) I_{2}(\tau-t) d t}{\left\langle I_{1}\right\rangle\left\langle I_{2}\right\rangle}
$$

clearly shows cross-correlation peak between lines, however its amplitude is small. CCF for the closest pair of lines is shown at fig. 4. 


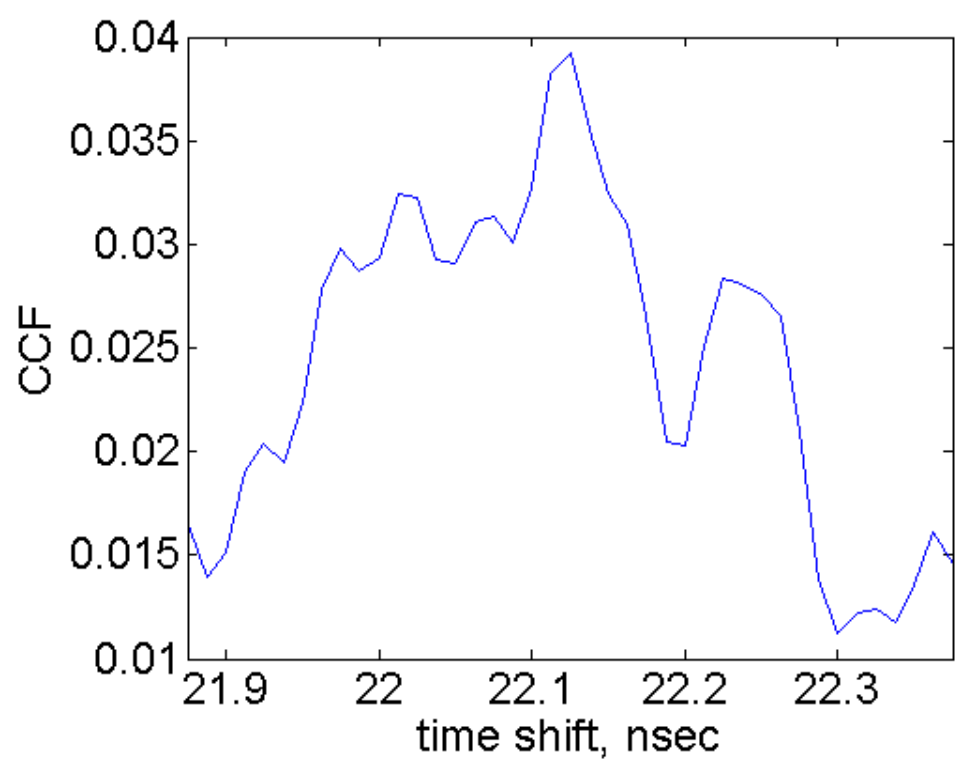

Figure 4. Cross-correlation function for nearest lines $(1555,82 \mathrm{~nm}$ and $1556.27 \mathrm{~nm})$.

Time shift between channels possibly arises due to different fiber length used after splitting signal at laser output. Wellpronounced peak indicates presence of correlations between two channels. Peak is significantly broadened, reason is not clear by now.

\section{CORRELATION ANALYSIS}

General characterization of two correlated traces can be provided with two-dimensional probability density function ( $2 \mathrm{~d}$ PDF), which characterizes probability of simultaneous observation of intensities $I_{1}$ and $I_{2}$ in channels 1 and 2 respectively. If correlation between traces is nonzero than $2 \mathrm{~d}$ PDF cannot be factorized to multiplication of PDFs of both traces:

$$
P\left(I_{1}, I_{2}\right) \neq P_{1}\left(I_{1}\right) \cdot P_{2}\left(I_{2}\right)
$$

To approximate 2d PDF a two-dimensional histogram was built: all range of intensity fluctuation was divided into 1024 bins for both channels, and all simultaneously measured intensity pairs were distributed over this $2 \mathrm{~d}$ matrix. As our buffer was $10^{9}$ points for $1024 \cdot 1024=1.048576 \cdot 10^{6}$ bins approximation was expected to be accurate. $2 \mathrm{~d}$ PDF for the nearest pair of lines is shown on fig. 5 


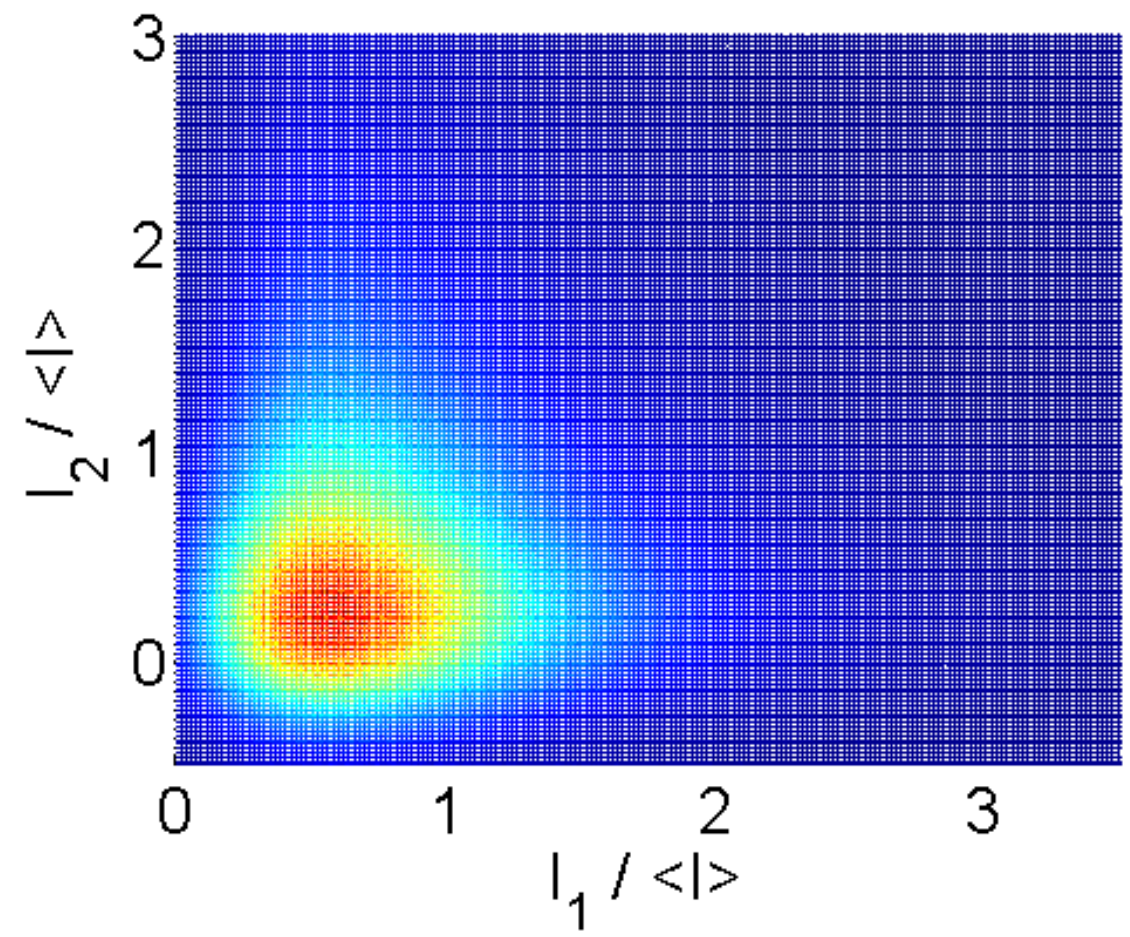

Figure 5. Two-dimensional probability for pair of closest lines (1555,82 nm and $1556.27 \mathrm{~nm})$.

Visually noticeable asymmetry relevant to line $I_{1}=I_{2}$ is the result of correlation of traces and arises from the fact that $2 \mathrm{~d}$ PDF cannot be factorized into production of one-dimensional probabilities.

Knowledge of 2d PDF allows calculation of distribution two-dimensional moments and cumulants:

$$
\begin{gathered}
\alpha_{n k}=\int I_{1}^{n} I_{2}^{k} P\left(I_{1}, I_{2}\right) d I_{1} d I_{2} \\
\kappa_{11}=\alpha_{11}-\alpha_{10} \alpha_{01}, \quad \kappa_{20}=\alpha_{20}-\alpha_{10}^{2}, \quad \kappa_{02}=\alpha_{02}-\alpha_{01}^{2}
\end{gathered}
$$

Correlation between two traces is often characterized by linear correlation coefficient (Pearson coefficient), varying between 1 and -1 :

$$
r=\frac{\kappa_{11}}{\sqrt{\kappa_{20} \kappa_{02}}}
$$

Pearson coefficient dependence on wavelength of second channel line is shown on fig. 6. 


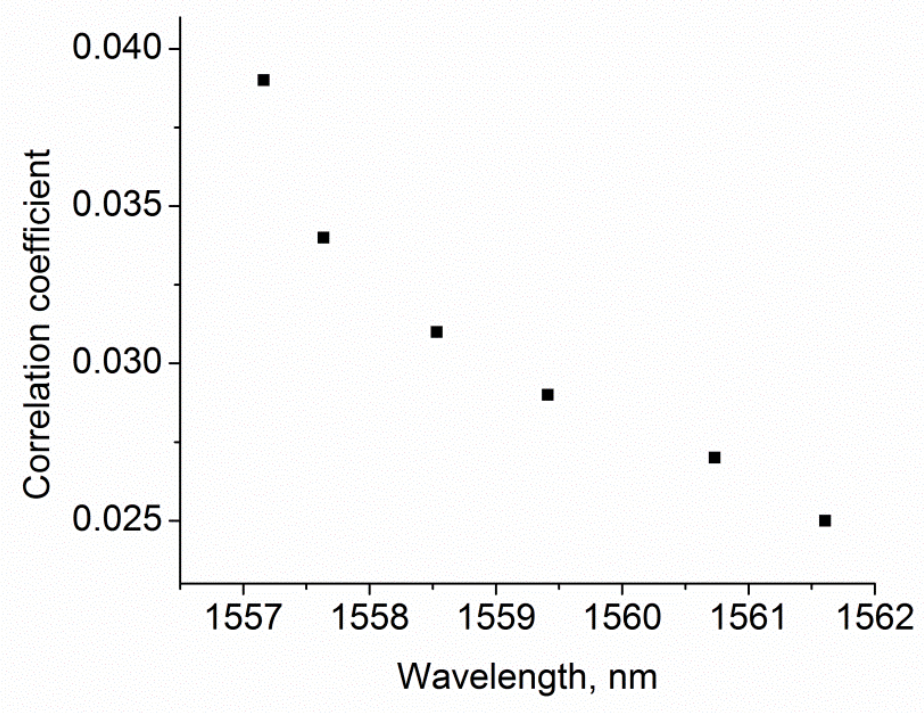

Figure 6. Pearson coefficient as function of spectral detuning.

Pearson coefficient is quite small - less than 0.1 - and decays with increase of separation between lines in spectrum. Decrease of cross-correlation for distant lines looks consistent, but note that even for highest spectral distance in our experiment - more than $6 \mathrm{~nm}$ - coefficient is the same order of magnitude as for the closest pair (spectral distance 0.9 $\mathrm{nm})$. Thus we can conclude that spectral correlations exist in radiation of RDFL with LF and can be detected by direct observation. Small qualitative values of correlation can be probably explained by big enough spectral width of lines; further search for cross-correlations is more convenient to perform with narrow spectral lines.

\section{CONCLUSION}

A direct simultaneous measurement of long time traces $\left(10^{9}\right.$ points $)$ representing two lines of spectra of fiber laser with randomly distributed feedback with Lyot filter was performed. Statistical correlation of the edge line and any other lines in spectrum, measured in experiment, are clearly visualized by peaks of cross-correlation function. Two-dimensional probability and conditional probability functions were calculated as histograms to visualize correlations. It was shown that Pearson correlation coefficient is quite small by value - about 0.03-0.04 - but is non-zero for all, even distat lines in spectrum.

\section{ACKNOWLEDGMENTS}

This work is supported by the Russian Foundation for Basic Research (16-32-00772).

\section{REFERENCES}

[1] R. Loudon, Quantum Theory of Light (OUP Oxford, 2000)

[2] D. V. Churkin, and S. V. Smirnov, "Numerical modelling of spectral, temporal and statistical properties of Raman fiber lasers," Opt. Commun. 285, 2154-2160 (2012).

[3] S. Randoux, and P. Suret, "Experimental evidence of extreme value statistics in Raman fiber lasers." Opt. Lett. 37, 500-502 (2012).

[4] A. E. Bednyakova, O. A. Gorbunov, M. O. Politko, S. I. Kablukov, S. V. Smirnov, D. V. Churkin, M. P. Fedoruk, and S. A. Babin, "Generation dynamics of the narrowband Yb-doped fiber laser," Opt. Express 21, 8177-8182 (2013).

[5] P. Walczak, S. Randoux, and P. Suret, "Statistics of a turbulent raman fiber laser," Opt. Lett. 40, 3101-3104 (2015). 
[6] O. A. Gorbunov, S. Sugavanam, and D. V. Churkin, "Intensity dynamics and statistical properties of random distributed feedback fiber laser," Opt. Lett. 40, 1783-1786 (2015)

[7] S. Sugavanam, Z. Yan, V. Kamynin, A. S. Kurkov, L. Zhang, and D. V. Churkin, "Multiwavelength generation in a random distributed feedback fiber laser using an all fiber lyot filter," Opt. Express 22, 2839-2844 (2014).

[8] Gorbunov O.A., Sugavanam S., Vatnik I.D., Churkin D.V., "Statistical properties of radiation of multiwavelength random DFB fiber laser", Optics Express, 24, 16, 19417-19423 (2016) 